\title{
Real-time monitoring system of bus seat belt based on the GPRS network module
}

\author{
Yeqiang $\mathrm{Lu}^{1, \mathrm{a}}$, Jingjing $\mathrm{GuO}^{2, \mathrm{~b}}$ and Hongyan $\mathrm{Chen}^{2, \mathrm{c}}$ \\ ${ }^{1}$ College of Youth Automotive, Hangzhou Vocational and Technical College, \\ Hangzhou,310018,China \\ ${ }^{2}$ College of Mechanical and Electrical Engineering, China Jiliang University, \\ Hangzhou,310018,China \\ a 13858112568@163.com, ${ }^{\mathrm{b}} 1462100265 @ q q . c o m,{ }^{\mathrm{c}} \mathrm{bbchy@163.com}$
}

Keywords: Si4432, GPRS network module, real-time monitoring, seat belt

\begin{abstract}
In view of the bus seat belt wearing not in place for the current, using modern detection technologies and single chip microcomputer (SCM) systems,designed real-time monitoring system of bus seat belt based on the GPRS network module.The information transmission between the bus data and the host is realized by Si4432 wireless data transceiver module. And then transmiting real-time data to the Passenger Transport Centre through the GPRS module. Compared to other monitoring systems, the system is excellent in response speed, transmission rate and production cost.
\end{abstract}

\section{Introduction}

Bus is as one of the three major options (rail, air and road )for travel, security issues of passenger has always been a considered problem of bus company and traffic management departments. Real-time monitoring system of bus seat belt based on the GPRS network module can reflect every passenger's seat belt lacing situation on the front driver's main screen. Therefore the driver can keep abreast of every passenger if wearing a seat belt, and then make the appropriate measures, Thus protecting the passengers in the car.[1,2] In addition, the system can also automatically sent passenger's attendance on the bus to Passenger Transport Centre monitoring terminal,so the staff can keep abreast of attendance. This paper analyzed the necessity of bus seat belt monitoring and the current problems, put forward the overall scheme of the system based on the GPRS network module, described each unit features, analyzed the communication protocol design and technical characteristics of the network nodes and so on.

\section{The overall scheme of the system based on the GPRS network module}

\subsection{Scheme architecture of bus seat belt real time monitoring system}

Real-time monitoring system of bus seat belt based on the GPRS network module mainly consist of the host system (data processing systems), the slave system (seat detection system) and PC systems (information monitoring system). The structure shown in Figure 1 


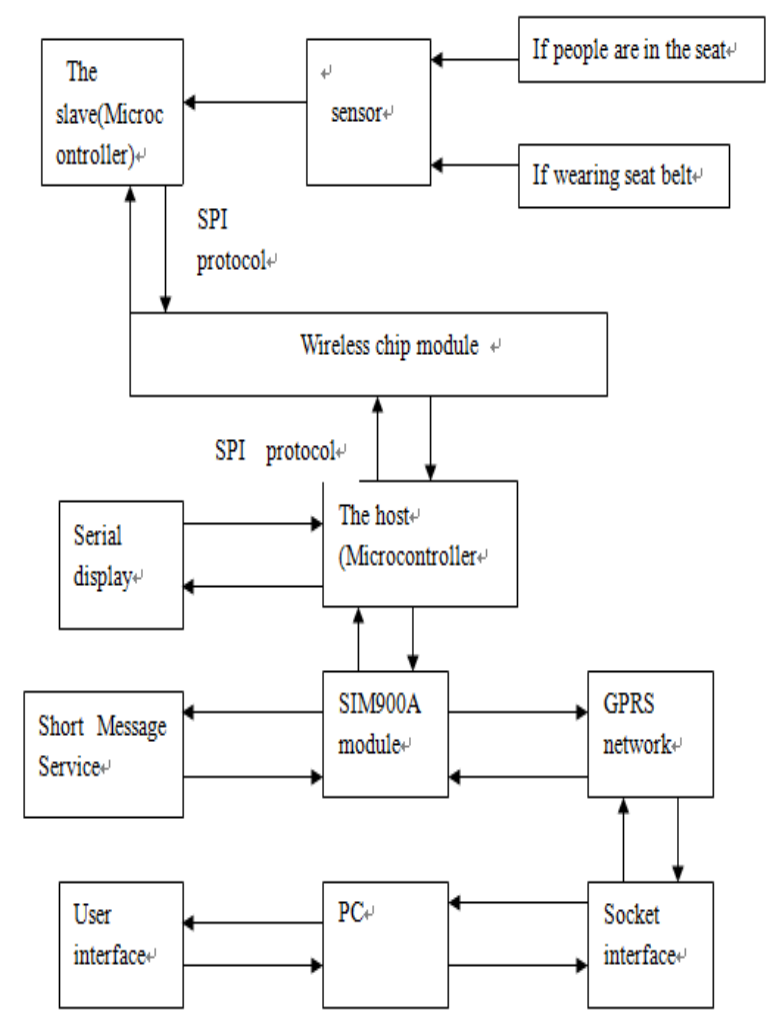

Figure 1 Overall system architecture

The slave system which consists of single chip microcomputer (including the main control chip, peripheral modules and power modules) and the sensor, can achieve the detection of seat state and transmit data to the host via SPI protocol.The host system which consists of single chip microcomputer (including the main control chip, peripheral modules and power modules)and serial display, can achieve the receiving and sending of GPRS network data and serial screen display and human-computer interaction.The PC system consists of a computer which connected to the external network IP.[3]The design tasks of vehicle terminal software are mainly in the following five part: wireless data transceiver module driver、GPRS driver、 data encryption algorithm、 human-computer interaction 、 data access.

\subsection{Operational principle of bus seat belt real time monitoring system}

The slave is responsible for monitoring the four states of seat in the bus:people seated and has seat belt buckled、people seated and has not seat belt buckled、no people and has seat belt buckled、 no people and has not seat belt buckled. Multiple slaves is organized, monitoring the status of all the seats in the bus. The host obtains the information from the slave through the wireless chip module,and gets all the states of seat, And then displays the status information on the serial display. When the states is no people and has seat belt buckled or no people and has not seat belt buckled,yellow is displayed;when the state is people seated and has seat belt buckled ,green is displayed; when the state is people seated and has not seat belt buckled,red is displayed and warning. The host obtains the position information of the bus through the SIM900A module.,And control the SIM900A module interact information with PC via the GPRS network,send the information of the wearing of seat belt and the location.The MCU controller of the slave gets the information that whether or not to wear the seat belt and seat by detecting the sensor.The MCU controller of the host obtains the state of each the slave through the wireless chip module, and obtains the data packets which sent from the slave, thus obtains the state of each seat of the bus. PC monitors the fixed IP address through the SocketAPI, the MCU controller of the host makes the SIM900A module connect to the above IP address through controlling the SIM900A module, so it achieves GPRS network communication.[4] 


\section{Communication protocol design and technical characteristics of the network nodes}

\subsection{Analysis of microcontroller circuit design}

Seat detection system uses STM8S208R8 for the MCU as the main control chip,it has the advantages of high reliability, low cost, strong interference and so on. The main characteristics of the STM8S208R8 chip are as follows:Operating voltage is 2.95V $~ 5.5 \mathrm{~V}$; CPU operating frequency is $24 \mathrm{MHz}$; Up to $128 \mathrm{~KB}$ of Flash, $6 \mathrm{~KB}$ of RAM, $2 \mathrm{~KB}$ of EEPROM; the transmission rate of CAN2.0B up to $1 \mathrm{Mb} / \mathrm{s}$; A more flexible clock selection;two UART and one SPI interfaces; one $\mathrm{I}^{2} \mathrm{C}$ interfaces. STM8S has an enhanced Harvard\&CISA architecture, three pipeline structure, When working in $24 \mathrm{M}$ crystal frequency, up to 20MBPS. It not only meets the requirements of processing speedand has a strong processing ability, but also has a rich on-chip peripherals, ease of use and modular design, effectively reduces the complexity of the system. It provides a good hardware platform for collecting data system development, fully meeting the requirements of the system.

\subsection{Analysis of the data processing system's hardware design}

Data processing system adopts the STM32F103VET6 of 32-bit MCU as the main control chip. The microcontroller's operating frequency is $72 \mathrm{MHz}$, high speed memory is integrated on chip, and connecting rich and enhanced peripherals andI/O through APB bus.There are two 12-bit ADC,one 12-bit two-channel DAC,eleven 16-bit timers,two $\mathrm{I}^{2} \mathrm{C}$ interfaces, three USART interfaces, two SPI interface, one CAN interface ,one USB interface and so on on the chip. Compared with the same type of microcontroller,it has the advantages of high integration, low power consumption, real time application, competitive price and so on.

\subsection{Transmission of GPS + GPRS module information}

GPS+GPRS module of this system uses the HUAWEI SIM900A module which has a good reputation. It has a three-band GSM/GPRS wireless module and convenient UART interface. UART interface communicates with the outside world through the serial and it is 3V voltage input and output. The way it works is that connects to a specified IP address via TCP / IP protocol , and communicates with the GPS module, obtains position information.[5] System and SIM900A module using serial communication, the system control SIM900A module to complete the short message, call and answer of telephone, GPRS network connection , receive and transmit data by sending a serial command,this makes the system realize wireless transmission and reception of data,and then making the system more flexible.The connection circuit of GPRS wireless communication module and STM32F103 serial are shown in Figure 2.

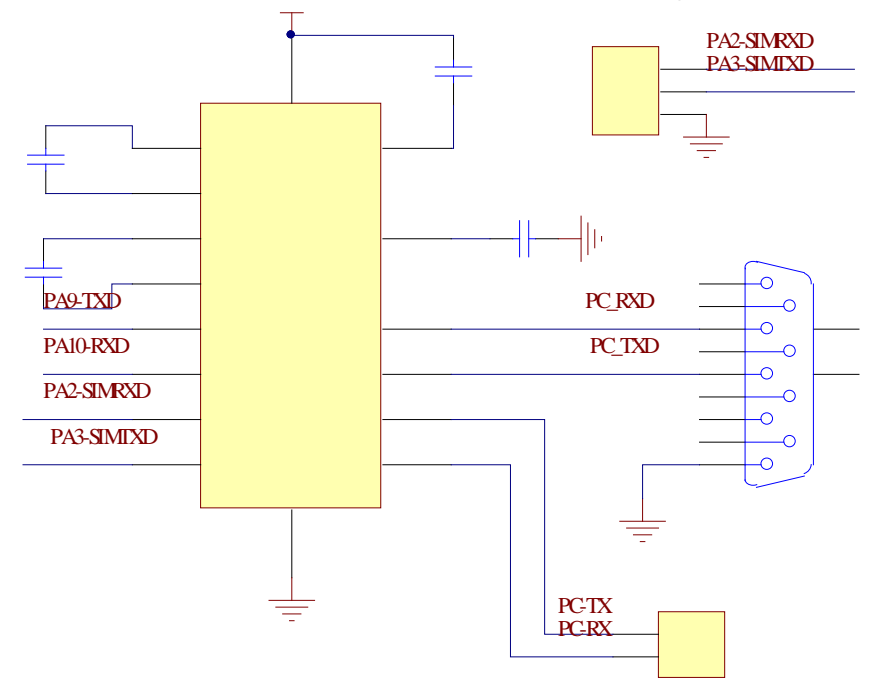

Figure 2 SIM900A serial connection circuit diagram

\subsection{Display module}

The graphic display module that selected is a module which SSD1963 is as the main control chip. This chip has $1215 \mathrm{k}$ buffer of byte frame to display and support $864 * 480 * 24$ bit graphic content; It is equipped with parallel interface bus of different widths to receive graphics data and commands 
the microcontroller; connection through the I/O port , namely four GPIO pins and built in clock generator, the background light can be controlled by pulse width modulation signal.

\section{Software design}

System's software consists of the main program, the wireless data transceiver module program, the network data transmission module program, the timer subroutine, delay subroutine and so on. The flow chart of main program are shown in Figure 3
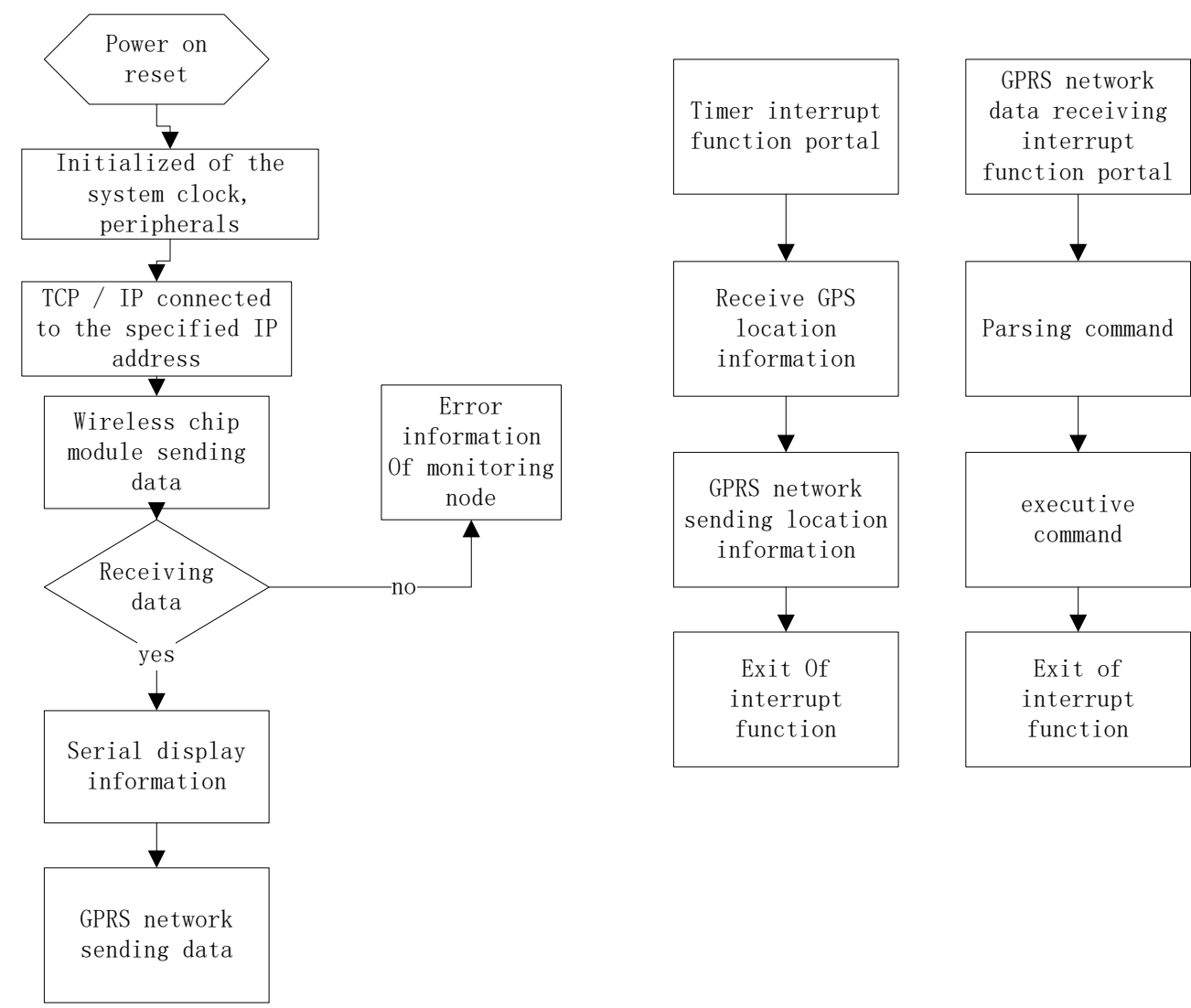

Figure 3 The flow chart of main program

\section{Summary}

In the background of the frequent accidents of bus ,it has an important meaning of designingthe real-time monitoring system of bus seat belt based on the GPRS network module. It can reflect every passenger's seat belt lacing situation on the driver's display,thus reminding the passengers who not wearing a seat belt to wear in time. According to the research content, the overall design scheme is established,the hardware system and software architecture of the vehicle terminal are built and the software of monitoring center platform is constructed. The system is in good condition by testing.

\section{Reference}

[1]Zhang Cheng. Seat belts- maintain the life[J] .Road traffic management.(2013)No.12,p56-56.

[2] Wang Xiaodong.Automobile collision and safety[M] .Tsinghua University Press.2000.p11-12

[3]Peng Jian,Ye Feng, Xin Jingze. Functional design and application of automobile remote monitoring diagnostic system.[J] Shanghai automobile, (2011)No.3,p27-28.

[4] Zhao Chunming, Qiao Xutong, Ma Ning,et al. Research on fault diagnosis of electric vehicle distributed control system based on CAN bus. [J] Vehicle and power technology, Vol, 98 (2005) No. 
2, p43-45.

[5] Gu Zongran, Meng Lifan, Yang Yanjun. Data acquisition and wireless transceiver system design.[J] Electronic testing. (2011)No.2,p69-70. 